\title{
THE SYNTACTIC NEAR-RING OF A LINEAR SEQUENTIAL MACHINE
}

\author{
by MICHAEL HOLCOMBE
}

(Received 5th March 1981)

\section{Linear sequential machines}

Let $R$ be a ring, a linear sequential machine over $R$ is a quintuple $\mathscr{M}=(Q, A, B, F, G)$ where

$$
\begin{aligned}
& Q, A, B \text { are } R \text {-modules, } \\
& F: Q \times A \rightarrow Q \text { and } G: Q \times A \rightarrow B \text { are } R \text {-homomorphisms. }
\end{aligned}
$$

We call $Q$ the set of states, $A$ the input alphabet and $B$ the output alphabet. Let $A^{*}, B^{*}$ be the free monoids generated by the sets $A, B$ respectively. The empty word $\Lambda$ will be regarded as a member of both $A^{*}$ and $B^{*}$. Let $x \in A^{*}$ we define a function $F_{x}: Q \rightarrow Q$ by

$$
\begin{aligned}
& q F_{\Lambda}=q \\
& q F_{x a}=F\left(q F_{x}, a\right) \quad \text { for } x \in A^{*}, \quad a \in A .
\end{aligned}
$$

The function $F_{x}$ will be called the next state function induced by $x$.

Now let $q \in Q$, we define a function

$$
\begin{aligned}
& f_{q}: A^{*} \rightarrow B^{*} \quad \text { by } \\
& f_{q}(\Lambda)=\Lambda \\
& f_{q}(x a)=f_{q}(x) f_{q F_{x}}(a) \text { for } x \in A^{*}, \quad a \in A .
\end{aligned}
$$

The function $f_{q}$ is the sequential function defined by $q$. (When $q=0$ we have the result, $f_{0}=f_{\mathscr{A}}$, of the machine as in Eilenberg [1]).

Now let $a \in A$, then for $q \in Q$

$$
\begin{aligned}
F(q, a) & =F(q, 0)+F(0, a) \\
& =q F_{0}+0 F_{a} .
\end{aligned}
$$

The function $F_{0}: Q \rightarrow Q$ is an $R$-endomorphism of $Q$ and we will write $0 F_{a}$ as $q_{a} \in Q$. 
Each state function $F_{a}: Q \rightarrow Q$ can thus be regarded as a sum of an $R$-endorphism $F_{0}$ and a constant function $\bar{q}_{a}: Q \rightarrow\left\{q_{a}\right\}$. The function $F_{a}$ is thus an affine function of $Q$. Now let $a, a^{\prime} \in A$ then

$$
\begin{aligned}
q F_{a a^{\prime}}=q F_{a} F_{a^{\prime}} & =\left(q F_{0}+q_{a}\right) F_{a^{\prime}} \\
& =\left(q F_{0}+q_{a}\right) F_{0}+q_{a^{\prime}} \\
& =q F_{0} F_{0}+q_{a} F_{0}+q_{a^{\prime}}
\end{aligned}
$$

which again is an affine function. Generally for $x \in A^{*}$ the function $F_{x}$ is an affine function of $Q$.

The set of all affine functions of $Q$, denoted by $\mathbf{M}_{\text {arr }}(Q)$, is a near-ring under the operation of addition and composition of functions (see Pilz [2]). It is natural to consider the syntactic monoid of $\mathscr{M}$, which is essentially the distinct state functions $F_{x}\left(x \in A^{*}\right)$, as a submonoid of $\mathbf{M}_{\text {arf }}(Q)$. This submonoid then generates, additively, a subnear-ring of $\mathbf{M}_{\text {arr }}(Q)$ and this will be called the syntactic near-ring of $\mathscr{M}$.

Before we proceed with our investigations we will consider some general properties of this type of near-ring.

\section{Affinely generated near-rings}

Let $(\Gamma,+)$ be any additive group and denote by End $\Gamma$ the semigroup of all endomorphisms of $\Gamma$. Let $\mathbf{M}(\Gamma)$ denote the set of all functions $f: \Gamma \rightarrow \Gamma$, and note that $\mathbf{M}(\Gamma)$ is a near-ring under the operations of mapping addition and composition. We may generate a near-ring $\mathbf{E}(\Gamma)$ which is the subnear-ring of $\mathbf{M}(\Gamma)$ generated by $\mathbf{E n d}(\Gamma)$ and it is seen that a typical element of $\mathbf{E}(\Gamma)$ is of the form $\sum_{i=1}^{n} \sigma_{i} e_{i}$ where $e_{i} \in \mathbf{E n d}(\Gamma)$ and $\sigma_{i}= \pm 1$. Such a near-ring, $\mathbf{E}(\Gamma)$ is called a distributively generated near-ring since the elements $e_{i} \in \mathbf{E n d}(\Gamma)$ are distributive elements of $\mathbf{M}(\Gamma)$ that generate $\mathbf{E}(\Gamma)$. This construction can be generalised by replacing End $(\Gamma)$ by any subsemigroup of $\operatorname{End}(\Gamma)$.

Another subset of $\mathbf{M}[\Gamma]$ is the set $\operatorname{Con}(\Gamma)$ of all constant functions $f: \Gamma \rightarrow \Gamma$, these are functions that satisfy $\gamma f=\gamma_{0}$ for all $\gamma \in \Gamma$, where $\gamma_{0}$ is a fixed element of $\Gamma$. The set Con $(\Gamma)$ is also a semigroup (under composition) in fact it is a near-ring. Furthermore, the following facts are immediate.

$$
\mathbf{M}(\Gamma) \cdot \operatorname{Con} \Gamma=\operatorname{Con} \Gamma=\operatorname{Con} \Gamma \cdot M(\Gamma)
$$

$$
\text { End } \Gamma+\operatorname{Con} \Gamma \text { is a subsemigroup of } M(\Gamma) \text {. }
$$

Now we consider forming the near-ring generated by the semigroup End $\Gamma+\operatorname{Con} \Gamma$. It is fairly clear that a typical element of this near-ring, $\mathbf{E C}(\Gamma)$, is of the form

$$
\sum_{i=1}^{n} \sigma_{i}\left(e_{i}+c_{i}\right) \quad \text { where } \quad \sigma_{i}= \pm 1, \quad e_{i} \in \operatorname{End} \Gamma, \quad c_{i} \in \operatorname{Con} \Gamma
$$

Furthermore in the case of $\Gamma$ an abelian group $\mathbf{M}_{\text {aff }}(\Gamma)=\mathbf{E C}(\Gamma)$. We will call $\mathbf{E C}(\Gamma)$ the 
affinely generated near-ring generated by $\operatorname{End} \Gamma+\operatorname{Con} \Gamma$. We can generalise this construction to a certain extent, e.g. by considering a subsemigroup of $\operatorname{End} \Gamma+\operatorname{Con} \Gamma$, or by looking at the situation in general near-rings.

Let $N$ be a near-ring, define

$$
\begin{gathered}
N_{0}=\{n \in N \mid 0 n=0\} \\
N_{c}=\{n \in N \mid 0 n=n\} \\
N_{d}=\left\{n \in N \mid\left(n_{1}+n_{2}\right) n=n_{1} n+n_{2} n, \forall n_{1}, n_{2} \in N\right\} .
\end{gathered}
$$

Then immediately $N_{c}=\left\{n \mid n_{1} n=n \forall n_{1} \in N\right\}$ since

$$
0 n=n \Rightarrow n_{1} n=n_{1} 0 n=0 n=n \text { for } n_{1} \in N .
$$

Both $N_{0}$ and $N_{c}$ are near-rings and $N_{d}$ is a semigroup. Also $N \cdot N_{\mathrm{c}}=N_{\mathrm{c}}=N_{\mathrm{c}} \cdot N$. The set $N_{d}+N_{c}$ is a semigroup. Suppose that $S$ is a subsemigroup of $N_{d}+N_{c}$. Define the set

$$
N_{S}=\left\{\sum_{i=1}^{n} \sigma_{i} s_{i} \mid \sigma_{i}= \pm 1, s_{i} \in S\right\}
$$

if $0 \in S$, we show that $N_{S}$ is a near-ring which clearly contains the semigroup $S$. Let $\sum_{i=1}^{n} \sigma_{i} s_{i}, \sum_{j=1}^{m} \sigma_{j}^{\prime} s_{j}^{\prime} \in N_{S}$ then clearly

and

$$
\sum_{i=1}^{n} \sigma_{i} s_{i}-\sum_{j=1}^{m} \sigma_{j}^{\prime} s_{j}^{\prime} \in N_{S}
$$

$$
\left(\sum_{i=1}^{n} \sigma_{i} s_{i}\right) \cdot\left(\sum_{j=1}^{m} \sigma_{j}^{\prime} s_{j}^{\prime}\right)=\sum_{j=1}^{m}\left(\sum_{i=1}^{n} \sigma_{i} s_{i}\right) \sigma_{j}^{\prime} s_{j}^{\prime} \in N_{S}
$$

Thus $N_{S}$ is the near-ring generated by $S$ and we call it the affinely generated (a.g.) nearring generated by $S . N_{S}$ is also a near-ring under other conditions on $S$.

Using a semigroup $S \subseteq N_{d}$ we see that the a.g. near-ring generated by $S$ is the d.g. near-ring generated by $S$ and so a.g. near-rings are generalisations of d.g. near-rings. We state some elementary consequences of the definition.

Proposition 1. Let $N$ be an a.g. near-ring generated by $S$ where $S$ is a subsemigroup of $N_{d}+N_{c}$.

(i) Each element of $N$ can be expressed in the form

$$
\sum_{i=1}^{n}\left(\sigma_{i} e_{i}+\sigma_{i}^{\prime} c_{i}\right) \quad \text { where } \quad \sigma_{i}, \sigma_{i}^{\prime}= \pm 1, \quad e_{i} \in N_{d}, \quad c_{i} \in N_{c} .
$$

(ii) If $K \subseteq N$ and $K N \subseteq K$ then $K\left(S \cap N_{d}\right)+\left(S \cap N_{c}\right) \subseteq K$, thus $S \cap N_{c} \subseteq K$.

(iii) A normal subgroup $K \subseteq N$ is a right ideal if $K N \subseteq K$. 


\section{The syntactic near-ring}

Returning to the linear sequential machine $\mathscr{M}=(Q, A, B, F, G)$ defined with respect to the ring $R$, we notice that the presence of an abelian group as the set $Q$ of states means that $\mathbf{M}_{\mathrm{ann}}(Q)$ is an a.g. near-ring. The syntactic near-ring generated by the syntactic monoid of $\mathscr{M}$ in the near-ring $\mathbf{M}_{\text {ant }}(Q)$ is an a.g. near-ring. Writing this as $N(\mathscr{M})$ we have $N(\mathscr{M})=N_{S}$ where $S$ is the syntactic monoid of $M$.

If we consider some of the properties of the transformation monoid $(Q, S)$ defined by the machine $\mathscr{M}$ we will obtain some indications about the kind of constructions that will be natural to consider for a.g. near-rings. The interplay between $\mathscr{M}$ and $N(\mathscr{M})$ may also be interesting. For example, $Q$ is naturally an $N(\mathscr{M})$-module since $Q$ is an abelian group and we may define

$$
q \cdot \sum_{i=1}^{n} \sigma_{i} s_{i}=\sum_{i=1}^{n} \sigma_{i}\left(q s_{i}\right) \in Q, \quad \sigma_{i}= \pm 1, \quad s_{i} \in S
$$

Furthermore for $x=a_{1} \ldots a_{k} \in A^{*}$ we have

$$
q F_{x}=q F_{0}^{k}+\sum_{i=1}^{k} q_{a_{i}} F_{0}^{k-i}=q\left(F_{0}^{k}+\sum_{i=1}^{k} \bar{q}_{a_{i}} F_{0}^{k-i}\right) .
$$

Let $\mathbf{Z}$ denote the ring of integers and let $\bar{Q}_{A}=\left\{\bar{q}_{a} \mid a \in A\right\}$ then $\bar{Q}_{A}$ is a subsemigroup of $\mathbf{M}_{\mathrm{an}}(Q)$.

Recall that $A$ is an abelian group and for $a, a^{\prime} \in A$ we have

$$
\bar{q}_{a}+\bar{q}_{a^{\prime}}=\bar{q}_{a+a^{\prime}}
$$

It can be easily established that $\bar{Q}_{A}$ is a near-ring.

Each $F_{x}$ corresponds to a type of polynomial and the syntactic near-ring $N_{s}$ may be considered to be the set of all polynomials of the form:

$$
f(\mathbf{x})+g(\mathbf{x}) \quad \text { where } \quad f(\mathbf{x}) \in \mathbf{Z}(\mathbf{x}), \quad g(\mathbf{x}) \in \bar{Q}_{A}(\mathbf{x}) .
$$

The correspondence is defined by noting that

$$
F_{x}=F_{0}^{k}+\sum_{i=1}^{k} \bar{q}_{a_{i}} F_{0}^{k-i} \leftrightarrow \mathbf{x}^{k}+\sum_{i=1}^{k} \bar{q}_{a_{i}} \mathbf{x}^{k-i}
$$

The near-ring $N(\mathscr{M})$ can thus be described as

$$
N(\mathscr{M})=\mathbf{Z}(\mathbf{x})+\bar{Q}_{\boldsymbol{A}}(\mathbf{x})
$$

Multiplication in $\mathbf{Z}(\mathbf{x})+\bar{Q}_{A}(\mathbf{x})$ is given by

$$
(f(\mathbf{x})+g(\mathbf{x})) \cdot\left(f^{\prime}(\mathbf{x})+g^{\prime}(\mathbf{x})\right)=f(\mathbf{x}) \cdot f^{\prime}(\mathbf{x})+g(\mathbf{x}) \cdot f^{\prime}(\mathbf{x})+g^{\prime}(\mathbf{x})
$$


where $f(\mathbf{x}) \cdot f^{\prime}(\mathbf{x})$ is the usual product in $\mathbf{Z}(\mathbf{x})$ and

$$
\begin{gathered}
g(\mathbf{x}) \cdot f^{\prime}(\mathbf{x})=\sum_{i=0}^{k} \sum_{j=0}^{l} n_{i} r_{j} \mathbf{x}^{j+1} \quad \text { where } \quad g(\mathbf{x})=\sum_{i=0}^{k} n_{i} x^{i} \in \bar{Q}_{A}(\mathbf{x}), \\
f^{\prime}(\mathbf{x})=\sum_{j=0}^{l} r_{j} \mathbf{x}^{j} \in \mathbf{Z}(\mathbf{x}), \quad f(\mathbf{x}) \in \mathbf{Z}(\mathbf{x}), \quad g^{\prime}(\mathbf{x}) \in \bar{Q}_{A}(\mathbf{x}) .
\end{gathered}
$$

Polynomials in $N(\mathscr{M})$ will be called syntactic polynomials. They are examples of more general polynomial constructions. For example let $R$ be a ring with identity, $N$ an abelian near-ring which is also an $R$-module.

If $R(\mathbf{x})$ is the usual polynomial ring and $N(\mathbf{x})$ is the near-ring of polynomials in $\mathbf{x}$ over $N$ under the multiplication

$$
g(\mathbf{x}) \cdot g^{\prime}(\mathbf{x})=g^{\prime}(\mathbf{x}) \quad \text { for } \quad g(\mathbf{x}), \quad g^{\prime}(\mathbf{x}) \in N(\mathbf{x})
$$

The set $R(\mathbf{x})+N(\mathbf{x})$ is a near-ring under the operations

$$
\begin{gathered}
f(\mathbf{x})+g(\mathbf{x})+f^{\prime}(\mathbf{x})+g^{\prime}(\mathbf{x})=f(\mathbf{x})+f^{\prime}(\mathbf{x})+g(\mathbf{x})+g^{\prime}(\mathbf{x}) \\
(f(\mathbf{x})+g(\mathbf{x})) \cdot\left(f^{\prime}(\mathbf{x})+g^{\prime}(x)\right)=f(\mathbf{x}) \cdot f^{\prime}(\mathbf{x})+g(\mathbf{x}) \cdot f^{\prime}(\mathbf{x})+g^{\prime}(\mathbf{x})
\end{gathered}
$$

with $g(\mathbf{x}) \cdot f^{\prime}(\mathbf{x})$ defined as in (3.6).

Let us denote this near-ring by $[R, N](\mathbf{x})$ and note that

$$
\left([R, N](\mathbf{x})_{d}=R(\mathbf{x})\right.
$$

and

$$
([R, N](\mathbf{x}))_{c}=N(\mathbf{x})
$$

Then $[R, N](\mathbf{x})$ is an a.g. near-ring.

Now we examine the output function $G: Q \times A \rightarrow B$. As before we have

$$
f_{q}(a)=q G_{a}=G(q, a)=q G_{0}+0 G_{a}
$$

and

$$
\begin{aligned}
f_{q}\left(a a^{\prime}\right) & =q G_{a} \cdot q F_{a} G_{a^{\prime}} \\
& =\left(q G_{0}+0 G_{a}\right)\left(q F_{0} G_{0}+q_{a} G_{0}+0 G_{a^{\prime}}\right)
\end{aligned}
$$

and so

$$
\begin{aligned}
f_{q}\left(a a^{\prime}\right) & =f_{q}(a) \cdot f_{q F_{a}}\left(a^{\prime}\right) \\
& =\left(f_{q}(0)+f_{0}(a)\right) \cdot\left(f_{q F_{0}}(0)+f_{q_{a}}(0)+f_{0}\left(a^{\prime}\right) \cdot\right) \\
& =f_{q}(a) \cdot\left(q F_{0} G_{0}+q_{a} G_{0}+0 G_{a}\right)
\end{aligned}
$$


and generally for $x=a_{1} \ldots a_{k} \in A^{*}$,

$$
f_{q}(x a)=f_{q}(x)\left(q F_{0}^{k} G_{0}+\sum_{i=1}^{k} q_{a_{i}} F^{k-i} G_{0}+0 G_{a}\right) .
$$

\section{Interrelations between $N(\mathscr{M})$ and $\mathscr{M}$}

Let $\mathscr{M}=(Q, A, B, F, G)$ and $\mathscr{M}^{\prime}=\left(Q^{\prime}, A, B, F^{\prime}, G^{\prime}\right)$ be linear sequential machines and consider a state function $\phi: Q \rightarrow Q^{\prime}$ satisfying

$$
\phi \text { is an } R \text {-module homomorphism, }
$$

and for $q \in Q, a \in A$,

$$
\begin{gathered}
\phi\left(q F_{a}\right)=\phi(q) F_{a}^{\prime} \\
\phi(q) G_{a}^{\prime}=q G_{a} .
\end{gathered}
$$

Theorem 4.2. If $\phi$ is a surjective state mapping then a near-ring homomorphism $\beta: N(\mathscr{M}) \rightarrow N\left(\mathscr{M}^{\prime}\right)$ exists such that, for $q \in Q, n \in N(\mathscr{M})$

$$
\phi(q n)=\phi(q) \beta(n)
$$

Furthermore for $q \in Q, x \in A^{*}, f_{q}(x)=f_{\phi(q)}^{\prime}(x)$.

Proof. Let $S$ and $S^{\prime}$ be the syntactic semigroups of $\mathscr{M}$ and $\mathscr{M}^{\prime}$ respectively. By a standard result in automata theory there exists a semigroup homomorphism $\gamma: S \rightarrow S^{\prime}$ such that $\phi(q s)=\phi(q) \gamma(s)$ for $q \in Q, s \in S$. Define $\beta: N(\mathscr{M}) \rightarrow N\left(\mathscr{M}^{\prime}\right)$ by

$$
\beta\left(\sum_{i=1}^{k} \sigma_{i} s_{i}\right)=\sum_{i=1}^{n} \sigma_{i} \gamma\left(s_{i}\right), \quad \sigma_{i}= \pm 1, \quad s_{i} \in S
$$

then clearly $\beta$ is a near-ring homomorphism. For $q \in Q, n=\sum_{i=1}^{k} \sigma_{i} s_{i} \in N(\mathscr{M})$ we have

$$
\begin{aligned}
\phi(q n) & =\phi\left(\sum_{i=1}^{k} \sigma_{i}\left(q s_{i}\right)\right)=\sum_{i=1}^{k} \sigma_{i} \phi\left(q s_{i}\right)=\sum_{i=1}^{k} \sigma_{i} \phi(q) \gamma\left(s_{i}\right) \\
& =\sum_{i=1}^{k} \phi(q) \sigma_{i} \gamma\left(s_{i}\right)=\phi(q) \sum_{i=1}^{k} \sigma_{i} \gamma\left(s_{i}\right)=\phi(q) \beta(n) .
\end{aligned}
$$

Now we show that $\phi: Q \rightarrow Q^{\prime}$ satisfies the condition

$$
f_{\phi(q)}^{\prime}=f_{q} \text { for } q \in Q
$$

Since, for $a \in A, f_{q}(a)=q G_{a}=\phi(q) G_{a}^{\prime}=f_{\phi(q)}^{\prime}(a)$ by (4.3) we can easily check that an 
inductive argument yields

$$
\begin{aligned}
f_{q}(x a) & =f_{q}(x) \cdot f_{q F_{x}}(x) \\
& =f_{\phi(q)}^{\prime}(x) \cdot f_{\phi\left(q F_{x}\right)}^{\prime}(a) \\
& =f_{\phi(q)}^{\prime}(x) \cdot f_{\phi(q) F_{x}^{\prime}}^{\prime}(a) \\
& =f_{\phi(q)}^{\prime}(x a)
\end{aligned}
$$

by a generalisation of (4.2) where $x \in A^{*}, \quad a \in A$.

Thus $f_{q}(x)=f_{\phi(q)}^{\prime}(x)$ for all $x \in A^{*}$.

Now we examine what happens when we consider the problem of minimising a machine $\mathscr{M}$.

Let $\mathscr{M}=(Q, A, B, F, G)$ be a linear sequential machine, we choose the zero of $Q$ as an initial state, and we are principally interested in realising the sequential function $f_{0}: A^{*} \rightarrow B^{*}$.

Define the relation $\sim$ on $Q$ by

$$
q \sim q^{\prime} \quad \text { iff } \quad f_{q}=f_{q^{\prime}} \quad\left(q, q^{\prime} \in Q\right)
$$

Theorem 4.3 For $q, q^{\prime} \in Q$

$$
q \sim q^{\prime} \quad \text { iff } \quad q F_{0}^{n} G_{0}=q^{\prime} F_{0}^{n} G_{0} \quad \text { for all } \quad n \geqq 0 .
$$

Proof. If $q \sim q^{\prime}$ then $f_{q}(x)=f_{q^{\prime}}(x)$ for all $x \in A^{*}$. Let $a \in A$ then $f_{q}(a)=q G_{a}=q G_{0}+0 G_{a}=$ $q^{\prime} G_{0}+0 G_{a}$ and so $q^{\prime} G_{0}=q G_{0}$.

Now assume that for words $x \in A^{*}$ of length less than $n$

$$
f_{q}(x)=f_{q^{\prime}}(x) \Rightarrow q F_{0}^{k} G_{0}=q^{\prime} F_{0}^{k} G_{0}, \quad 0 \leqq k<n .
$$

Then

$$
f_{q}(x a)=f_{q^{\prime}}(x a) \Rightarrow f_{q F_{x}}(a)=f_{q^{\prime} F_{x}}(a)
$$

and so $q F_{x} G_{a}=q^{\prime} F_{x} G_{a}$. Now

$$
\begin{aligned}
q F_{x} G_{a} & =\left(q F_{0}^{n}+\sum_{i=1}^{n} q_{a_{i}} F_{0}^{n-i}\right) G_{a} \\
& =\left(q F_{0}^{n}+\sum_{i=1}^{n} q_{a_{i}} F^{n-i}\right) G_{0}+0 G_{a}=q F_{0}^{n} G_{0}+\sum_{i=1}^{n} q_{a_{i}} F_{0}^{n-i} G_{0}+0 G_{a}
\end{aligned}
$$




$$
\begin{aligned}
q^{\prime} F_{x} G_{a} & =\left(q^{\prime} F_{0}^{n}+\sum_{i=1}^{n} q_{a_{i}} F_{0}^{n-i}\right) G_{0}+0 G_{a} \\
& =q^{\prime} F_{0}^{n} G_{0}+\sum_{i=1}^{n} q_{a_{i}} F_{0}^{n-i} G_{0}+0 G_{a}
\end{aligned}
$$

where $x=a_{1} \ldots a_{n}$.

Thus $q F_{0}^{n} G_{0}=q^{\prime} F_{0}^{n} G_{0}$ and so we have established the first part of the theorem.

The converse is proved similarly.

Theorem 4.4. Let $R=\{q \in Q \mid q \sim 0\}$ then $R$ is an $N$-submodule of $Q$.

Proof. Clearly $\sim$ is an equivalence relation. Now let $q \sim q^{\prime}, q_{1} \sim q_{1}^{\prime}$ and consider $f_{q-q_{1}}$ and $f_{q^{\prime}-q_{1}}$, then for $a \in A$,

$$
\begin{aligned}
f_{q-q_{1}}(a) & =\left(q-q_{1}\right) G_{a}=\left(q-q_{1}\right) G_{0}+0 G_{a} \\
& =q G_{0}-q_{1} G_{0}+0 G_{a}=q^{\prime} G_{0}-q_{1}^{\prime} G_{0}+0 G_{a} \\
& =f_{q^{\prime}-q_{1}^{\prime}}(a) .
\end{aligned}
$$

Assume that $f_{q-q_{1}}(x)=f_{q^{\prime}-q_{1}^{\prime}}(x)$ for all $x \in A^{*}$ of length less than or equal to $n$ and consider

$$
f_{q-q_{1}}(x a) \quad \text { where } x a \text { is of length } n+1 \text {. }
$$

Then

$$
f_{q-q_{1}}(x a)=f_{q-q_{1}}(x) \cdot f_{\left(q-q_{1}\right) F_{x}}(a)=f_{q^{\prime}-q_{1}^{\prime}}(x) \cdot f_{\left(q-q_{1}\right) F_{x}}(a)
$$

Now

$$
\begin{aligned}
f_{\left(q-q_{1}\right) F_{x}}(a) & =\left(q-q_{1}\right) F_{x} G_{a} \\
& =\left(q-q_{1}\right)\left(F_{0}^{n}+\sum_{i=1}^{n} q_{a_{i}} F_{0}^{n-i}\right) G_{0}+0 G_{a} \\
& =\left(q F_{0}^{n}-q_{1} F_{0}^{n}+\sum_{i=1}^{n} q_{a_{i}} F_{0}^{n-i}\right) G_{0}+0 G_{a} \\
& =q F_{0}^{n} G_{0}-q_{1} F_{0}^{n} G_{0}+\sum_{i=1}^{n} q_{a_{i}} F_{0}^{n-i} G_{0}+0 G_{a} \\
& =q^{\prime} F_{0}^{n} G_{0}-q_{1}^{\prime} F_{0}^{n} G_{0}+\sum_{i=1}^{n} q_{a_{i}} F_{0}^{n-i} G_{0}+0 G_{a} \\
& =f_{\left(q^{\prime}-q_{1}^{\prime}\right) F_{x}}(a), \text { where } x=a_{1} \ldots a_{n} \text { by Theorem 4.3. }
\end{aligned}
$$

Thus $q-q_{1} \sim q^{\prime}-q_{1}^{\prime}$ and so $R$ is a subgroup of $Q$. 
Finally for $n \in N$ we have $n=\sum_{i=1}^{k} \sigma_{i} s_{i}, \sigma_{i}= \pm 1, s_{i} \in S$. Then if $q \sim q^{\prime}$ we have $q n=$ $\sum_{i=1}^{k} \sigma_{i} q s_{i}, q^{\prime} n=\sum_{i=1}^{k} \sigma_{i} q^{\prime} s_{i}$ and we now show that $q n \sim q^{\prime} n$. For this we note that if $q s_{i} \sim q^{\prime} s_{i}$ then $q n \sim q^{\prime} n$. So we must establish that $x \in A^{*}, q F_{x} \sim q^{\prime} F_{x}$. For $a \in A$,

$$
f_{q}(x a)=f_{q}(x) f_{q F_{x}}(a)=f_{q^{\prime}}(x) f_{q^{\prime} F_{x}}(a)=f_{q}(x) f_{q^{\prime} F_{x}}(a)
$$

and so

$$
f_{q F_{x}}(a)=f_{q^{\prime} F_{x}}(a)
$$

Now let $f_{q F_{x}}(y)=f_{q F_{x}}(y)$ for all words $y \in A^{*}$ of length $n$ or less. Let $y$ be of length $n$, and consider

$$
\begin{aligned}
f_{q F_{x}}(y a) & =f_{q F_{x}}(y) f_{q F_{x y}}(a)=f_{q^{\prime} F_{x}}(y) f_{q F_{x y}}(a) \\
& =f_{q^{\prime} F_{x}}(y) f_{q^{\prime} F_{x y}}(a)=f_{q^{\prime} F_{x}}(y a) .
\end{aligned}
$$

Hence $q F_{x} \sim q^{\prime} F_{x}$. This completes the proof (because of Proposition 1(iii)).

A linear sequential machine $\mathscr{M}=(Q, A, B, F, G)$ is called accessible if given any $q \in Q$ there exists $x \in A^{*}$ such that $0 F_{x}=q$. This clearly means that any state is reachable from the initial state 0 . As far as the $N$-module $Q$ goes this means that 0 is a generator, that is

$$
0 \cdot N \supseteq 0 \cdot S=Q \text { and so } Q=0 \cdot N=0 \cdot N_{c} \text {. }
$$

A machine $\mathscr{M}$ is called reduced if the relation defined in (4.5) is trivial, that is $q \sim q^{\prime} \Rightarrow$ $q=q^{\prime}$ for all $q, q^{\prime} \in Q$.

An accessible, reduced linear machine $\mathscr{M}$ is called minimal. Given a general linear machine $\mathscr{H}=(Q, A, B, F, G)$ we can obtain a minimal machine with the same behaviour by forming the accessible part of $\mathscr{M}$, this is the machine $\mathscr{M}^{a}$ with state set $Q^{\prime}=Q \cdot S$ replacing the set $Q$ and the induced state and output maps. The accessible machine $\mathscr{M}^{a}$ is reduced by forming the quotient machine $\mathscr{M}^{a} / \sim$ in the usual way. Since $Q^{\prime}$ is an $R$ module it is clear that $Q^{\prime}=Q \cdot N$. The minimal machines of $\mathscr{M}$ are essentially unique, up to isomorphism. (Eilenberg [1] Chapter XVII.)

We now prove

Theorem 6.5. Let $\mathscr{M}=(Q, A, B, F, G)$ be a reduced machine. Then $Q$ has no proper non-zero $N$-submodules $K$ satisfying $K G_{0}=\{0\}$.

Proof. Let $K \subseteq Q$ be an $N$-subgroup, then $K$ is a subgroup of $Q$ and $K \cdot N \subseteq K$. Let $\sim_{K}$ be a relation defined on $Q$ by

$$
q \sim_{K} q^{\prime} \Leftrightarrow q-q^{\prime} \in K, \quad\left(q, q^{\prime} \in Q\right) .
$$


Choose $q, q^{\prime}$ such that $q \sim{ }_{K} q^{\prime}$ then there exists a $k \in K$ with $q^{\prime}=q \dot{+}$.

Now, for $a \in A$,

$$
f_{q^{\prime}}(a)=f_{q+k}(a)=q G_{0}+k G_{0}+0 G_{a}=q G_{0}+0 G_{a}=f_{q}(a)
$$

Let $x \in A^{*}$ and suppose that $f_{q^{\prime}}(x)=f_{q}(x)$ for all $x$ of length less than or equal to $n$. Now let $x a \in A^{*}$ be of length $n+1$, then

$$
f_{q^{\prime}}(x a)=f_{q^{\prime}}(x) f_{q^{\prime} F_{x}}(a)=f_{q}(x) f_{(q+k) F_{x}}(a)
$$

Now $(q+k) F_{x}-q F_{x} \in K$ and so $(q+k) F_{x}=q F_{x}+k^{\prime}$ for some $k^{\prime} \in K$. Then

$$
\begin{aligned}
f_{q^{\prime}}(x a) & =f_{q}(x)\left(q F_{x} G_{0}+k^{\prime} G_{0}+0 G_{a}\right) \\
& =f_{q}(x)\left(q F_{x} G_{0}+0 G_{a}\right) \\
& =f_{q}(x) f_{q F_{x}}(a)=f_{q}(x a)
\end{aligned}
$$

Hence we have $q=q^{\prime}$ since $M$ is reduced and $K=\{0\}$.

Finally we combine this last result with the accessibility condition to obtain:

Theorem 4.6. Let $\mathscr{M}=(Q, A, B, F, G)$ be a minimal linear sequential machine. Then the $N$-module $Q$ satisfies:

(i) $Q$ possesses no proper non-zero $N$-submodules $K$ such that $K G_{0}=\{0\}$

(ii) $Q$ is generated by 0 .

Further properties of the syntactic near-ring of a linear sequential machine will be examined in a forthcoming paper.

\section{REFERENCES}

1. S. Ellenberg, Automata, Languages and Machines, Vol. A (Academic Press, New York, 1974).

2. G. Pilz, Near-Rings (North-Holland, Amsterdam, 1977).

Department of Pure Mathematics

QUEEN'S UNIVERSITY

BELFAST BT7 INN 